\title{
Myocardial extracellular volume fraction quantification in an animal model of the doxorubicin-induced myocardial fibrosis: a synthetic hematocrit method using $3 T$ cardiac magnetic resonance
}

\author{
Zhen Zhou ${ }^{1}$, Rui Wang ${ }^{1}$, Hui Wang ${ }^{1}$, Yi Liu ${ }^{1}$, Dongxu Lu ${ }^{1}$, Zhonghua Sun ${ }^{2}$, Guang Yang ${ }^{3,4}$, Lei Xu ${ }^{1}$ \\ ${ }^{1}$ Department of Radiology, Beijing Anzhen Hospital, Capital Medical University, Beijing, China; ${ }^{2}$ Department of Medical Radiation Sciences, Curtin \\ University, Perth, WA, Australia; ${ }^{3}$ Cardiovascular Research Centre, Royal Brompton Hospital, London, UK; ${ }^{4}$ National Heart and Lung Institute, \\ Imperial College London, London, UK
}

Correspondence to: Lei Xu, MD. Department of Radiology, Beijing Anzhen Hospital, Capital Medical University, 2 Anzhen Rd, Chaoyang District, Beijing 100029, China. Email: leixu2001@hotmail.com.

Background: Visualization of diffuse myocardial fibrosis is challenging and mainly relies on histology. Cardiac magnetic resonance (CMR), which uses extracellular contrast agents, is a rapidly developing technique for measuring the extracellular volume (ECV). The objective of this study was to evaluate the feasibility of the synthetic myocardial ECV fraction based on 3.0 T CMR compared with the conventional ECV fraction.

Methods: This study was approved by the local animal care and ethics committee. Fifteen beagle models with diffuse myocardial fibrosis, including 12 experimental and three control subjects, were generated by injecting doxorubicin $30 \mathrm{mg} / \mathrm{m}^{2}$ intravenously every three weeks for 24 weeks. Short-axis (SAX) and 4-chamber long-axis (LAX) T1 maps were acquired for both groups. The association between hematocrit (Hct) and native $\mathrm{T} 1_{\text {blood }}$ was derived from 9 non-contrast CMR T1 maps of 3 control beagles using regression analysis. Synthetic ECV was then calculated using the synthetic Hct and compared with conventional ECV at baseline and the $16^{\text {th }}$ and $24^{\text {th }}$ week after doxorubicin administration. The collagen volume fraction (CVF) value was measured on digital biopsy samples. Bland-Altman plots were used to analyze the agreement between conventional and synthetic ECV. Correlation analyses were performed to explore the association among conventional ECV, synthetic ECV, CVF, and left ventricular ejection fraction (LVEF).

Results: The regression model synthetic Hct $=816.46^{*} \mathrm{R} 1_{\text {blood }}-0.01\left(\mathrm{R}^{2}=0.617 ; \mathrm{P}=0.012\right)$ was used to predict the Hct from native $\mathrm{T} 1_{\text {blood }}$ values. The conventional and synthetic ECV fractions of experimental animals at the $16^{\text {th }}$ and $24^{\text {th }}$ week after modeling were significantly higher than those measured at the baseline $(31.4 \% \pm 2.2 \%$ and $36.3 \% \pm 2.1 \%$ vs. $22.9 \% \pm 1.7 \% ; 29.9 \% \pm 2.4 \%$ and $36.1 \% \pm 2.6 \%$ vs. $22.0 \% \pm 2.4 \%$; all with $\mathrm{P}<0.05)$. Bland-Altman plots showed a bias $(1.0 \%)$ between conventional and synthetic ECV with $95 \%$ limits of agreement of $-2.5 \%$ to $4.4 \%$ in the per-subject analysis $(n=21)$ and a bias $(1.0 \%)$ between conventional and synthetic ECV with $95 \%$ limits of agreement of $-2.4 \%$ to $4.3 \%$ in the per-segment analysis $(n=294)$. Conventional and synthetic ECV were well correlated with CVF ( $r=0.937$ and 0.925 , all with $\mathrm{P}<0.001$, $\mathrm{n}=10)$.

Conclusions: Our study showed promising results for using synthetic ECV compared with the conventional ECV for providing accurate quantification of myocardial ECV without the need for blood sampling.

Keywords: Cardiac magnetic resonance imaging (CMR imaging); diffuse interstitial myocardial fibrosis; extracellular volume fraction (ECV fraction); collagen volume fraction (CVF); correlation 
Submitted Mar 27, 2020. Accepted for publication Sep 18, 2020.

doi: 10.21037/qims-20-501

View this article at: http://dx.doi.org/10.21037/qims-20-501

\section{Introduction}

Myocardial fibrosis is a common end-point in a wide range of pathological processes affecting heart muscle (1). It can present in the form of focal fibrotic scars caused by myocyte death (apoptosis, autophagy, or necrosis) or as diffuse fibrosis due to expansion of the collagen fiber network around individual myocytes or myocyte bundles $(2,3)$.

Cardiovascular magnetic resonance (CMR) with late gadolinium enhancement (LGE) is an established gold standard noninvasive technique for evaluating focal fibrosis, featuring high spatial resolution, high contrast-to-noise ratio, and possible whole heart coverage using threedimensional (3D) scanning sequences; CMR has also begun to be used for prognostic evaluation $(3,4)$. Nevertheless, there is a high demand for the development of new techniques to quantify diffuse fibrosis better. Recently, CMR T1 mapping with extracellular volume (ECV) fraction estimation has shown the potential for detecting and quantifying both focal and diffuse changes in myocardial structure. This technique is increasingly used to identify the etiology for a wide range of cardiomyopathies (5-11).

Myocardial ECV fraction has become increasingly associated with diffuse myocardial fibrosis and is emerging as a marker for myocardial tissue remodeling that can provide a physiologically intuitive unit of measurement $(12,13)$. However, conventional methods for ECV quantification require blood hematocrit (Hct) samples and laboratory analysis, the inconvenience of which may pose a barrier for easy clinical application (14).

In this study, we explored the relationship between the longitudinal relaxation of blood and blood Hct based on 3T CMR and then generated an immediate synthetic ECV calculation without blood draws. The performance of the synthetic ECV fraction was evaluated and compared with the conventional ECV fraction based on $3 \mathrm{~T}$ CMR. Additionally, we investigated the impact of slice position and orientation on the ECV measurements owing to the current recommendations on T1 mapping and ECV, which suggested adding a long axis map to aid the analysis (15). The objective of this study was to evaluate the feasibility of noninvasive synthetic myocardial ECV fraction quantification of doxorubicin-induced myocardial fibrosis based on 3T CMR compared with the conventional ECV fraction.

\section{Methods}

\section{Experimental set-up and research subjects}

The detailed animal experiment flow is shown in Figure 1. This study was approved by the Local Animal Care and Use Committee. Fifteen female beagles were prospectively selected and randomly assigned to the experimental group $(n=12)$ and control group $(n=3)$. All beagles underwent CMR before doxorubicin administration. Then, 3 of the 12 experimental animals were sacrificed for pre-modeling histological analysis. The remaining experimental $(\mathrm{n}=9)$ and control dogs $(n=3)$ received doxorubicin (doxorubicin hydrochloride, Cayman Chemical, Ann Arbor, MI, USA) $30 \mathrm{mg} / \mathrm{m}^{2}$ body surface area intravenously, every 3 weeks, until a cumulative dose of $240 \mathrm{mg} / \mathrm{m}^{2}$ body surface area was reached (16). Unfortunately, 2 of the experimental dogs died from acute heart failure during the period of modeling, and all of their data (including pretreatment images) were excluded from the statistical analysis. After 16 weeks of modeling, the remaining subjects $(\mathrm{n}=10)$ underwent $\mathrm{CMR}$, and 3 experimental subjects were sacrificed for histological biopsy. The remaining experimental $(\mathrm{n}=4)$ and control dogs $(\mathrm{n}=3)$ were continued with myocardial fibrosis modeling and underwent CMR again at the $24^{\text {th }}$ week. Finally, all remaining experimental beagles $(n=4)$ were sacrificed for histological biopsy.

\section{CMR examination preparation}

Anesthesia was performed using an intramuscular injection of a mixture of xylazine hydrochloride $(2.0 \mathrm{mg} / \mathrm{kg}$, Rompun, Bayer, Seoul, Korea) and ketamine hydrochloride (1.0 mg/kg, Hospira, Lake Forest, CA, USA). Sublingual metoprolol (1.25 mg/kg, AstraZeneca, London, UK) was given to reduce the heart rate. Before CMR, the forelimb vein of each beagle was prepared for intravenous contrast agent injection, and blood was drawn for laboratory Hct analysis. The electrocardiogram (ECG) electrodes were placed on the chest of the beagles, and the dogs were free- 


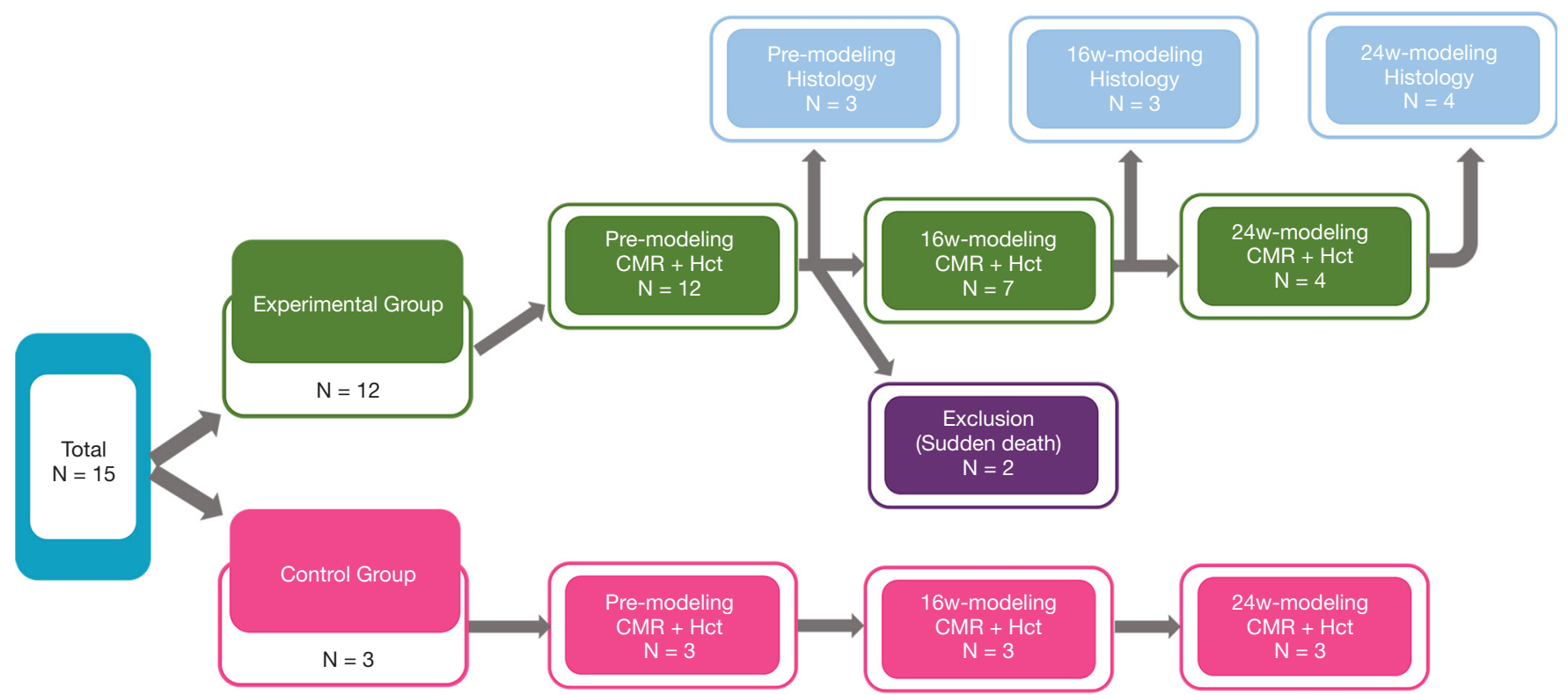

Figure 1 Flow chart of the animal studies.

breathing during the CMR scanning.

\section{CMR imaging protocol}

The CMR images were obtained using a 3T MRI scanner (Verio, Siemens, Erlangen, Germany) using a 32-channel cardiovascular array coil (InVivo, Orlando, FL, USA). Cine, native, and post-contrast T1-mapping images were acquired together with the LGE CMR. Steady-state free precession (SSFP) cine sequences were acquired in shortaxis (SAX) views to analyze cardiac function. The scanning parameters included retrospective ECG gating, repetition time $(\mathrm{TR})=45.6 \mathrm{~ms}$, echo time $(\mathrm{TE})=1.66 \mathrm{~ms}$, a field of view $(\mathrm{FOV})=219 \mathrm{~mm} \times 260 \mathrm{~mm}$, matrix $=256 \times 256$ pixels, slice thickness $=6 \mathrm{~mm}$, spatial resolution $=1.0 \times 0.9 \mathrm{~mm}^{2}$, and flip angle $=50^{\circ}$. An 11-heart-beat modified Look-Locker sequence with inversion recovery [MOLLI, 5(3)3 scheme, SSFP readout] was used for the CMR T1 measurement, as described previously (14). The T1-mapping acquisition of a 4-chamber long-axis (4-ch LAX) view and 3 SAX views were performed before and $15 \mathrm{~min}$ after the gadopentetate dimeglumine injection $(0.15 \mathrm{mmol} / \mathrm{kg})$ at a rate of $2.0 \mathrm{~mL} / \mathrm{s}$ followed by a $10 \mathrm{~mL}$ saline flush. The parameters used were retrospective ECG gating, TR $=351.2 \mathrm{~ms}$, TE $=1.14 \mathrm{~ms}$, FOV $=222 \mathrm{~mm} \times 260 \mathrm{~mm}$, slice thickness $=4 \mathrm{~mm}$ for the $4-\mathrm{ch}$ LAX images and $6 \mathrm{~mm}$ for the SAX views, matrix $=150 \times 192$ pixels, resulting in a spatial resolution of $1.7 \times 1.2 \mathrm{~mm}^{2}$, and flip angle $=35^{\circ}$. Using the phase-sensitive inversion recovery prepared SSFP sequence, LGE images of 4-ch LAX and SAX views were performed 8 min after the contrast agent injection. The parameters included retrospective ECG gating, $\mathrm{TR}=577 \mathrm{~ms}, \mathrm{TE}=1.67 \mathrm{~ms}, \mathrm{FOV}=211 \mathrm{~mm} \times$ $260 \mathrm{~mm}$, matrix $=156 \times 256$ pixels, slice thickness was as the same as the T1 mapping scanning, spatial resolution $=1.6 \times 1.0 \mathrm{~mm}^{2}$, flip angle $=20^{\circ}$, and average acquisition time was about $10 \mathrm{~s}$ per slice.

\section{CMR image quality control}

Quality evaluation of all CMR images and image postprocessing were performed independently by 2 observers (each with $>5$ years of experience in CMR image interpretation), who were blinded to the clinical data. Based on visual assessment of image artifacts and boundary sharpness between the myocardium and cavity of the left ventricle (LV), the image quality of T1 maps were classified as good (no artifacts with clear boundary, score $=3$ ), adequate (minimal or moderate artifacts, score $=2$ ), or poor (severe artifacts, score $=1$ ).

To extract myocardial T1 values, regions of interests (ROIs) for signal intensity measurement were drawn automatically on the myocardium as well as in the blood pool of the SAX view using cvi42 software (Circle Cardiovascular Imaging, Calgary, AB, Canada) 


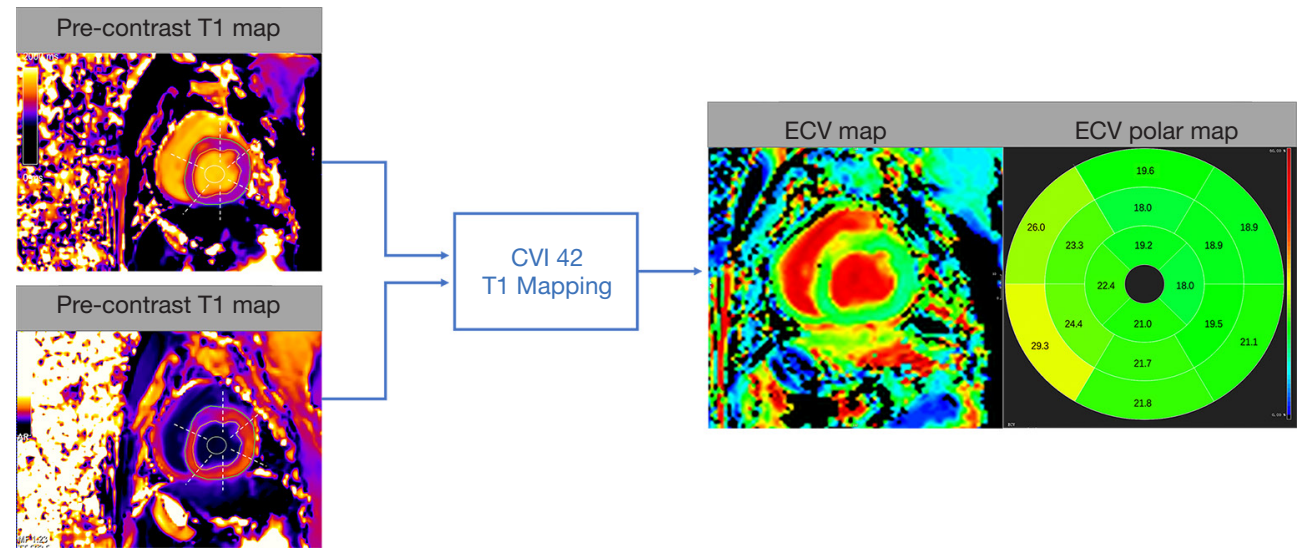

Figure 2 Process of generating the ECV maps. ECV map and ECV polar map of the AHA were generated automatically from pre-contrast T1 maps and post-contrast T1 maps using dedicated software (Circle Cardiovascular Imaging, CVI42). ECV, extracellular volume; AHA, American Heart Association.

(Figure 2). We also drew ROIs in myocardium and blood pool at the intersections of SAX and 4-ch LAX views to compare the native $\mathrm{T} 1$ and $\mathrm{ECV}$ values from the SAX view against those extracted from the corresponding LAX view. The ROIs were placed at the center of the myocardial wall (the midmural was above $10 \%$ from the endocardial and epicardial borders) carefully to avoid signal contamination from adjacent blood. None of the study subjects had focal LGE in the ROIs.

Myocardial ECV was then calculated using the following equation,

$$
\mathrm{ECV}=\left(\frac{1}{\mathrm{~T} 1_{\text {post-myo }}}-\frac{1}{\mathrm{~T} 1_{\text {pre-myo }}}\right) \div\left(\frac{1}{\mathrm{~T} 1_{\text {post-blood }}}-\frac{1}{\mathrm{~T} 1_{\text {pre-blood }}}\right) \times(1-\mathrm{Hct})
$$

where $T 1_{\text {pre-myo }}$ and $T 1_{\text {post-myo }}$ denoted the $\mathrm{T} 1$ values of myocardium before and after administration of the Gadolinium contrast agent (GBCA), and $\mathrm{T} 1_{\text {pre-blood }}$ and $\mathrm{T} 1_{\text {post-blood }}$ were the corresponding $\mathrm{T} 1$ values of the blood (17). Function and volumes were measured from SAX views of the $L V$ using the cvi42 software.

\section{Synthetic hematocrit and ECV}

A linear relationship between the longitudinal relaxivity ( $\mathrm{R} 1=1 / \mathrm{T} 1)$ of blood and lab measured Hct has been found in prior studies and can be determined by the water fractions of plasma and the erythrocyte cytoplasm (18-20). Therefore, a regression model could be used to predict the Hct from $\mathrm{T} 1_{\text {blood }}$ values using native $\mathrm{T} 1$ maps of the 3 control beagles with the corresponding laboratory blood
Hct. Native $T 1_{\text {blood }}$ values were measured from $S A X$ views of the midventricular LV. Synthetic Hct $\left(\right.$ Hct $\left._{\text {synthetic }}\right)$ was then obtained using the equation describing the linear regression between native $\mathrm{T} 1_{\text {blood }}$ and lab measured Hct. Conventional Hct was substituted by the synthetic Hct to derive the synthetic ECV

$\mathrm{ECV}_{\text {Synthetic }}=\left(\frac{1}{\mathrm{~T} 1_{\text {post-myo }}}-\frac{1}{\mathrm{~T} 1_{\text {pre-myo }}}\right) \div\left(\frac{1}{\mathrm{~T} 1_{\text {post-blood }}}-\frac{1}{\mathrm{~T} 1_{\text {pre-blood }}}\right) \times\left(1-\mathrm{Hct}_{\text {synthetic }}\right)$

Then we compared synthetic ECV to the conventional ECV with laboratory blood Hct and correlated them with the reference standard of the histological CVF.

\section{Histologic analysis}

After CMR examination, specimens from the middle LV septum segments of the experimental beagles were collected and fixed in $10 \%$ formalin. And then these specimens were dehydrated with alcohol in increasing concentration and embed in paraffin wax. After these processes, sectioning was performed. The corresponding part at the SAX plane of the midventricular LV level was selected using a slice thickness of $5 \mu \mathrm{m}$ followed by Masson staining. Quantitative analyses were conducted on digital biopsy samples using QuantCenter 2.1 software (3DHISTECH, Budapest, Hungary). To calculate the CVF, ROIs were manually drawn in a Masson-stained slice corresponding to the CMR ROI areas in the midventricular LV septum myocardium; we then calculated the average CVF value, which was taken as a surrogate of the dog's global CVF value. Two 
Table 1 Baseline characteristics of the beagles in each experimental group

\begin{tabular}{lccc}
\hline Characteristics & Pre-modeling subjects $(\mathrm{n}=10)$ & 16-week models $(\mathrm{n}=7)$ & 24-week models $(\mathrm{n}=4)$ \\
\hline Weight $(\mathrm{kg})$ & $8.3 \pm 0.9$ & $7.1 \pm 1.0^{*}$ & $9.1 \pm 0.8$ \\
Sex & Female & Female & Female \\
Heart rate (beats/min) & $99 \pm 6$ & $83 \pm 7$ & $82 \pm 10$ \\
Hematocrit (\%) & $49.1 \pm 1.5$ & $45.9 \pm 1.3$ & $43.9 \pm 1.6^{\#}$ \\
CVF $(\%)$ & $3.6 \pm 0.8(\mathrm{n}=3)$ & $23.1 \pm 1.7(\mathrm{n}=3)^{*}$ & $30.5 \pm 2.7(\mathrm{n}=4)^{\#}$ \\
LV systolic function & & & $26.7 \pm 0.9$ \\
EDV $(\mathrm{mL})$ & $24.1 \pm 1.9$ & $26.6 \pm 0.8^{*}$ & $15.3 \pm 0.5^{\#}$ \\
ESV $(\mathrm{mL})$ & $11.5 \pm 1.2$ & $14.0 \pm 0.7^{*}$ & $11.4 \pm 0.8$ \\
SV $(\mathrm{mL})$ & $12.6 \pm 1.6$ & $12.5 \pm 0.7$ & $42.8 \pm 1.7$ \\
LVEF $(\%)$ & $52.1 \pm 4.3$ & $47.1 \pm 2.1$ &
\end{tabular}

Values are given as mean $\pm \mathrm{SD}$. *, 16-week models vs. pre-modeling subjects, $\mathrm{P}<0.05$. \#, 24-week models vs. pre-modeling subjects, $\mathrm{P}<0.05$. CVF, collagen volume fraction; LV, left ventricle; EDV, end-diastolic volume; ESV, end-systolic volume; SV, stroke volume; LVEF, left ventricular ejection fraction.

pathologists analyzed the histological data with 5 years of experience.

\section{Statistical analyses}

Statistical analyses were performed using SPSS (version 25.0, IBM Corp., Chicago, IL, USA) and MedCalc (version 19, Ostend, Belgium). Continuous data with a normal distribution were presented as mean and standard deviation $($ mean $\pm \mathrm{SD})$. Categorical variables were described as frequencies or percentages. Bland-Altman plots were used to analyze the agreements among conventional ECV, synthetic ECV, CVF values, and the agreement between native $\mathrm{T} 1_{\text {myo }}$ and $\mathrm{ECV}$ values derived from the SAX views and corresponding LAX views. Pearson correlation analysis was used to determine the association among native $\mathrm{T} 1_{\text {myo }}$, conventional $\mathrm{ECV}$, synthetic $\mathrm{ECV}, \mathrm{CVF}$, Hct, and LV ejection fraction (LVEF). The significance of the differences between the 2 correlation coefficients was tested using the Fisher r-to-z transformation. The changes in CVF were evaluated using a one-way analysis of variance (ANOVA), while the variations in native $T 1_{\text {myo }}$, conventional ECV, and synthetic ECV were evaluated using repeated ANOVA. Inter-observer agreement was assessed using the intraclass correlation coefficient (ICC). A $\mathrm{P}$ value of $<0.05$ was considered statistically significant.

\section{Results}

\section{Characteristics of the studied subjects in the experimental group}

Table 1 shows the characteristics of the subjects in the experimental group. The CVF and end-systolic volume (ESV) of experimental subjects increased during the modeling, while Hct decreased (all $\mathrm{P}<0.05$ ). In the persubject analysis, both conventional and synthetic ECV was inversely related to the Hct $(\mathrm{n}=21$, including 10 subjects at pre-modeling, 7 subjects at the $16^{\text {th }}$ week, and 4 subjects at the $24^{\text {th }}$ week, $r=-0.814$ and -0.768 , all $\mathrm{P}<0.001)$ and LVEF ( $\mathrm{n}=21, \mathrm{r}=-0.801$ and -0.738 , all with $\mathrm{P}<0.001)$.

\section{Quality of the CMR T1-mapping images}

We analyzed a total of 336 SAX segments and 126 LAX segments of the LV. Of these, 404 segments $(87.4 \%$, SAX $\mathrm{n}=294$, LAX $\mathrm{n}=110$ ) satisfied the diagnostic requirements. Results showed that 300 segments $(64.9 \%)$ were good (score $=3), 104$ segments $(22.5 \%)$ were adequate (score $=2$ ), and 58 segments $(12.6 \%)$ were poor (score $=1)$. The poor segments, which might have been due to respiratory motion and could influence the accuracy of measurements, were thus excluded from further image analysis. 


\section{CMR ECV analysis}

The association between conventional Hct and native $\mathrm{T} 1_{\text {blood }}$ was derived from 9 non-contrast CMR T1 mappings of the control subjects. The linear regression equation was derived as: synthetic Hct $=816.46 \times R 1-0.01$ with $R^{2}=0.617$, and $\mathrm{P}=0.012$, where $\mathrm{R} 1$ represents the native $1 / \mathrm{T} 1$ value of the blood (Figure 3).

After doxorubicin injection, the ECV values increased and were significantly different among groups (conventional ECV: $22.9 \% \pm 1.7 \%$ for the pre-modeling group, $31.4 \% \pm 2.2 \%$ for the 16 th-week group, and $36.3 \% \pm 2.1 \%$ for the 24th-week group; corresponding synthetic ECV: $22.0 \% \pm 2.4 \%, 29.9 \% \pm 2.4 \%$, and $36.1 \% \pm 2.6 \%$; all $\mathrm{P}<0.05, \mathrm{n}=294$ in per-segment analysis). The laboratory Hct correlated well with the synthetic Hct across all

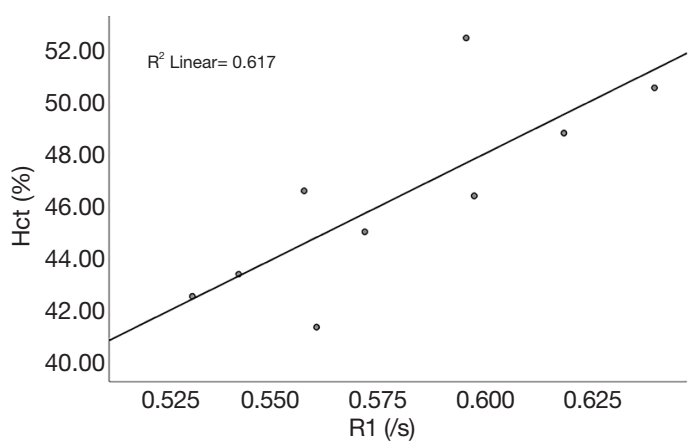

Figure 3 Correlation R1 of blood vs. Hct. The regression line between hematocrit (Hct) and pre-contrast R1 of the blood was linear with a $\mathrm{R}^{2}=0.617, \mathrm{P}<0.001$. the analyzed subjects $(\mathrm{n}=21 ; \mathrm{r}=0.599 ; \mathrm{P}<0.05)$. A good correlation was found between conventional ECV and synthetic ECV across all the analyzed segments $(n=294$; $\mathrm{r}=0.959$; $\mathrm{P}<0.001)$. Bland-Altman plots showed a bias (1.0\%) between conventional and synthetic ECV with $95 \%$ limits of agreement from $-2.5 \%$ to $4.4 \%$ in the per-subject analysis $(\mathrm{n}=21)$ and a bias $(1.0 \%)$ between conventional and synthetic ECV with $95 \%$ limits of agreement from $-2.4 \%$ to $4.3 \%$ in the per-segment analysis $(n=294)$ (Figure 4$)$. The correlation coefficient for the inter-observer agreement of conventional ECV calculation was high [ICC $=0.958(95 \%$ CI: $0.947-0.966)]$. The native $\mathrm{T} 1_{\text {myo }}$ values of the $16^{\text {th }}$-week and $24^{\text {th }}$-week groups were significantly higher than those obtained from the pre-modeling group $(1,175.1 \pm 7.0 \mathrm{~ms}$, $1,207.9 \pm 5.0 \mathrm{~ms}$, and $1,229.8 \pm 4.8 \mathrm{~ms}$ for the groups of premodeling, $16^{\text {th }}$ week and $24^{\text {th }}$ week, $\mathrm{P}<0.001, \mathrm{n}=192$ in persegment analysis).

There were no significant differences in native $\mathrm{T} 1_{\text {myo }}$ and $\mathrm{ECV}$ values between SAX and 4-ch LAX acquisitions using MOLLI (all $\mathrm{P}>0.05, \mathrm{n}=110$ in per-segment analysis). Bland-Altman plots showed a bias (0.4 ms) and 95\% limits of agreement (-16.4 to $17.1 \mathrm{~ms}$ ) for native $\mathrm{T} 1_{\text {myo }}$ values and a bias $(-0.1 \%)$ and $95 \%$ limits of agreement $(-2.3 \%$ to $2.0 \%)$ for ECV values between SAX and 4-ch LAX acquisitions (Figure 5; n=110 in per-segment analysis). There was no specific focal LGE in all of the analyzed segments.

\section{Histology analysis}

Figure 6 shows the histological results of typical beagles in the experimental groups. The myocardial cells of the pre-

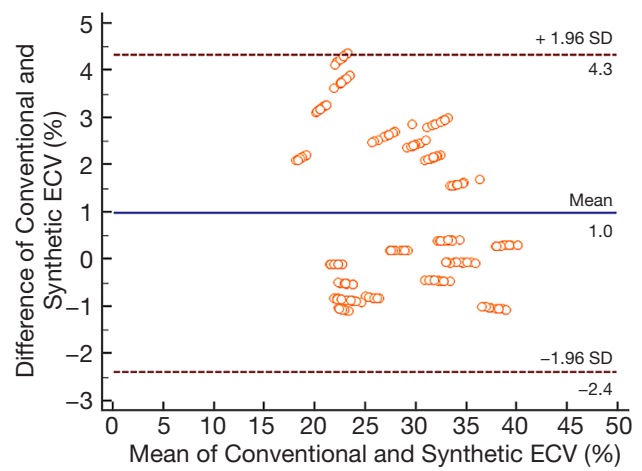

Figure 4 Validation of synthetic ECV vs. conventional ECV. Bland-Altman plots showed a small bias (1.0\%) between conventional and synthetic ECV with $95 \%$ limits of agreement from $-2.5 \%$ to $4.4 \%$ in per-subject analysis ( $\mathrm{n}=21$; left) and a small bias (1.0\%) between conventional and synthetic ECV with $95 \%$ limits of agreement from $-2.4 \%$ to $4.3 \%$ in per-segment analysis (n=294; right). ECV, extracellular volume. 

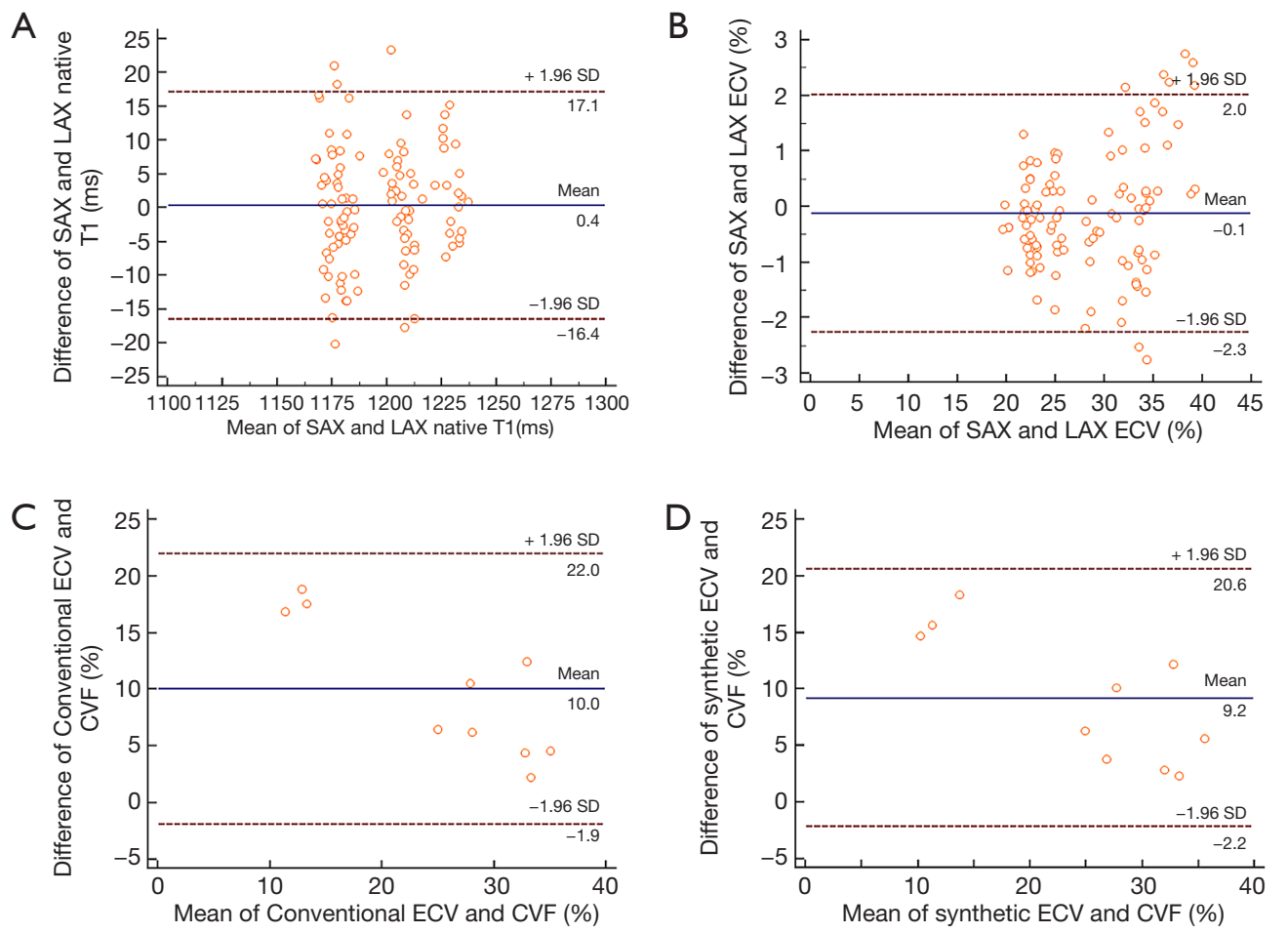

Figure $5 \mathrm{SAX} v s$. LAX acquisitions using MOLLI and conventional and synthetic ECV vs. CVF. In per-segment analysis (n=110), there were no significant differences found in native T1 with a bias of $0.4 \mathrm{~ms}$ and $95 \%$ limits of agreement from -16.4 to $17.1 \mathrm{~ms}(\mathrm{~A})$ and no significant differences found in ECV values with a bias of $-0.1 \%$ and $95 \%$ limits of agreement from $-2.3 \%$ to $2.0 \%$ (B). Bland-Altman plots showed a bias (10.0\%) between conventional ECV and CVF with 95\% limits of agreement from $-1.9 \%$ to $22.0 \%$ (C) and a bias (9.2\%) between synthetic ECV and CVF with $95 \%$ limits of agreement from $-2.2 \%$ to $20.6 \%$ (D) in per-subject analysis (n=10). SAX, short-axis; LAX, long-axis; ECV, extracellular volume; CVF, collagen volume fraction.
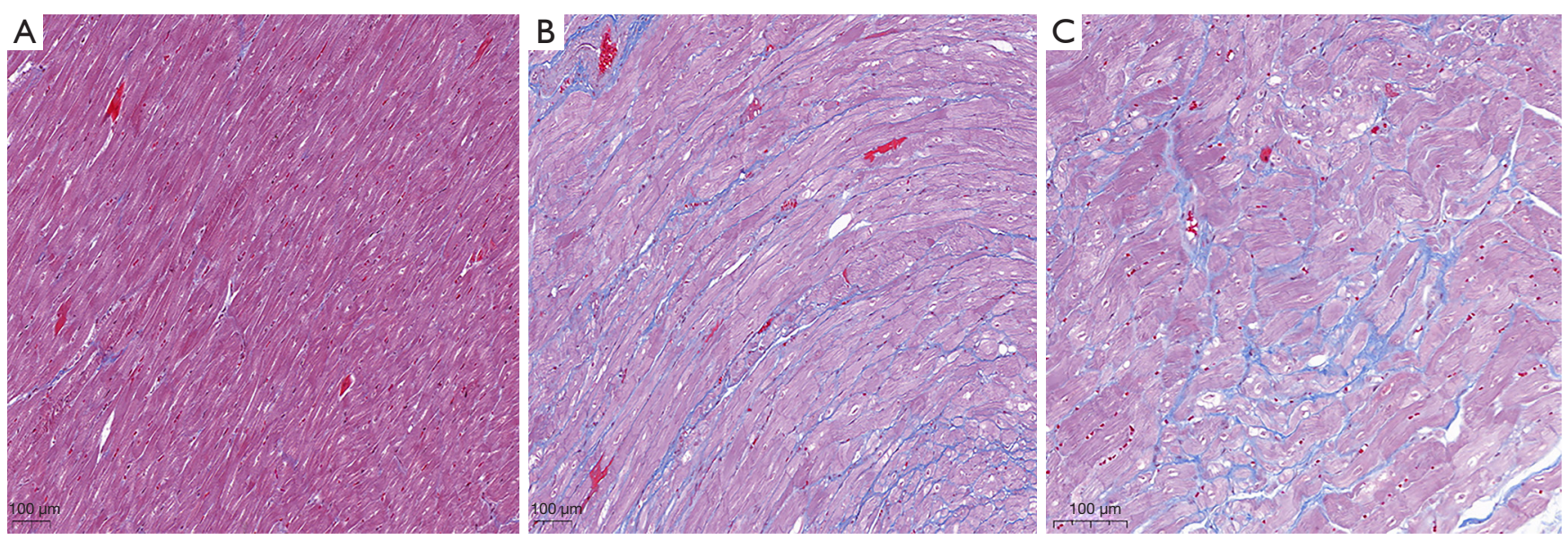

Figure 6 Example histological samples at the pre-modeling stage (A), 16th week (B), and 24th week (C). Masson's trichrome staining (blue, fibrosis; purple, myocardial fibers; original magnification $\times 100$ ). Histological studies showed that with an increased duration of modeling time, diffuse interstitial fibrosis became increasingly serious and collagen volume fraction values were increased. 
modeling subjects were normal and orderly, while irregular arrangements were observed in the modeling groups. Both conventional ECV and synthetic ECV correlated well with the histological CVF results ( $\mathrm{r}=0.937$ for the conventional $\mathrm{ECV} ; \mathrm{r}=0.925$ for the synthetic $\mathrm{ECV}$; all $\mathrm{P}<0.001 ; \mathrm{n}=10$ in per-subject analysis) and did not reach a significant difference using Fisher r-to-z transformation $(\mathrm{z}=0.17$, $\mathrm{P}=0.865)$. Bland-Altman plots showed a bias $(10.0 \%)$ between conventional ECV and CVF with $95 \%$ limits of agreement from $-1.9 \%$ to $22.0 \%$ and a bias $(9.2 \%)$ between synthetic ECV and CVF with $95 \%$ limits of agreement from $-2.2 \%$ to $20.6 \%$ (Figure $5 C, D$ ).

\section{Discussion}

This study aimed to explore the feasibility of synthetic ECV fraction based on $3 \mathrm{~T}$ CMR compared with conventional CMR ECV fraction in assessing doxorubicin-induced diffuse interstitial myocardial fibrosis. Additionally, we verified the consistency of SAX native $\mathrm{T}_{\text {myo }}$ and $\mathrm{ECV}$ values against those extracted from the corresponding LAX image as recommended by the Society for Cardiovascular Magnetic Resonance (SCMR) (15).

The ECV fraction, as derived from the ratio of T1 signal values, can provide a noninvasive tool for the quantification of tissue alterations in myocardial disease, and represents a physiological parameter. The ECV may also be more reproducible using different field strengths, vendors, and acquisition techniques than native and post-contrast $\mathrm{T} 1$ values (14).

Currently, the capability of using CMR T1 mapping along with ECV measurement to detect diffuse myocardial fibrosis has been validated in a wide range of cardiovascular diseases (CVDs), including acute and chronic myocardial infarction (AMI and CMI) $(21,22)$, chronic aortic regurgitation (23), heart failure (HF) (24), dilated cardiomyopathy (DCM) (25), and hypertrophic cardiomyopathy (HCM) (26). Sado et al. reported native T1 values in AMI, HCM, DCM, severe aortic stenosis (AS), and cardiac immunoglobulin light chain amyloidosis (AL) amyloidosis were high, and ECV values of AMI were among the highest of all these CVDs. They also revealed that CMR ECV could be a potentially useful marker for CVDs (27). In a separate study, Radunski et al. performed a comprehensive comparison of the accuracy in diagnosing acute myocarditis by conventional CMR techniques and novel mapping techniques. They found that native T1 and ECV of severe myocarditis (new-onset HF or acute chest pain) were raised. They also showed a better diagnostic accuracy using T1 mapping and in particular using the ECV (5). Thus, previous studies have demonstrated the potential of quantifying myocardial fibrosis using CMR T1 mapping and conventional ECV.

However, conventional ECV measurement is complicated, requiring venous blood sampling. The timeconsuming nature of venipuncture is the major obstacle for deploying this technique in actual clinical practice; therefore, simplification of ECV measurement is in demand. Treibel et al. sought to generate a synthetic ECV fraction using the relationship between Hct and longitudinal relaxation rate of blood-based on $1.5 \mathrm{~T}$ CMR (28). They found that the synthetic ECV correlated well with conventional ECV in participants with a wide range of health complaints and CVDs (including hypertrophic cardiomyopathy, severe aortic stenosis, and cardiac amyloidosis). In another study, Robison et al. evaluated the diagnostic accuracy of using ECV determined from laboratory Hct (Lab-ECV), a noninvasive point-of-care Hct device (POC-ECV), and synthetic Hct (synthetic$\mathrm{ECV}$ ). They also found no significant differences among Lab-ECV, POC-ECV, and synthetic-ECV (29). Most of the previous studies on synthetic ECV were based on the use of $1.5 \mathrm{~T}$ CMR, while few studies have evaluated the feasibility of synthetic ECV based on 3T CMR, especially for chemotherapy-related cardiomyopathy. Replication of studies would be required to evaluate the feasibility of this synthetic method for both field strengths to accommodate the inherent difference in $\mathrm{T} 1$ values between $1.5 \mathrm{~T}$ and $3 \mathrm{~T}$ scanners.

Considering the immaturity of this synthetic method, we evaluated the diffuse myocardial fibrosis in doxorubicininduced beagles using $3 \mathrm{~T}$ CMR. Anthracyclines, a broad spectrum of the anti-tumor drug, is widely used in the treatment of malignant tumors but have the potential to cause diffuse myocardial fibrosis and adverse cardiac events (30). The beagle heart is more significant in size with a thicker myocardium compared to the other commonly used experimental animals, for example, rabbits. The beagle's heart rate (HR) is also slower compared to rabbits, and that may reduce motion artifacts and therefore provide more accurate validation of the ECV calculation. Also, we used the simplified technique to calculate ECV without serologic analysis, i.e., utilizing the relationship between laboratory Hct and native $\mathrm{T} 1_{\text {blood }}$ values to derive a synthetic Hct for immediate synthetic ECV calculation. Compelling results have shown the promise of using the synthetic ECV 
technique, but the reliability of synthetic ECV derived from $3 \mathrm{~T} \mathrm{CMR}$ in patients receiving doxorubicin treatment requires further verification.

Although the $\mathrm{T} 1_{\text {blood }}$-based synthetic Hct and ECV technique may be a convenient and concise research tool, the studies of Shang et al. on 3T CMR and Raucci et al. on 1.5 T CMR revealed that ECV calculated from synthetic Hct can lead to significant miscategorization for individual patients with subtly elevated ECV, children, and young adults $(31,32)$. Further, before undergoing a CMR scan, most patients would require venipuncture for the gadolinium IV insertion, so access to venous blood may rarely be a problem. Hematocrit tests are simple, inexpensive, and fast, which raises the question of whether it should ever be omitted. Thus, it is advised that synthetic ECV be used only in clinical or research settings where laboratory Hct cannot be obtained. The leading utility of this method may be at follow up, serial examinations for those exposed to cardiotoxic medications such as doxorubicin, who require long-term serial evaluations to monitor signs of early cardiotoxicity. It is essential to interpret results based on the synthetic method scrupulously.

This study had some limitations. Firstly, the sample size was small in both the experimental and control groups, and the robustness of the synthetic method may need further validation using a large dataset. The regression model predicting Hct from native T1-blood values was derived from the native T1 maps of just 3 control beagles with a contemporaneous paired laboratory Hct. This may not be statistically robust, and we did not test the accuracy of this regression model in other animal cohorts. Besides, only one MOLLI sequence was evaluated; further studies are needed to assess the synthetic ECV derived by estimating Hct from native $T 1_{\text {blood }}$ values acquired with other commonly used CMR sequences, such as ShMOLLI, SASHA, ANGIE, saturation preparation (AIR), or a combination of MOLLI and SASHA (SAPPHIRE) (15). Moreover, further research is required to carefully explore the efficacy of this synthetic ECV method in a broader range of CVDs.

\section{Conclusions}

In conclusion, synthetic Hct derived from the regression of blood Hct and native $\mathrm{T} 1_{\text {blood }}$ values allows accurate quantification of the myocardial ECV fraction without drawing blood in a beagle model of doxorubicin-induced myocardial fibrosis. The synthetic ECV method can offer an alternative option for myocardial tissue characterization. Although this study has shown promising results from using synthetic ECV, further multicenter and multiscanner studies are necessary to verify the reliability and reproducibility of this synthetic ECV method.

\section{Acknowledgments}

We are immensely grateful to the imaging technology team in our hospital for their help and support.

Funding: This work was supported by grants from the National Key R\&D Program of China (2016YFC1300300) and the National Natural Science Foundation of China (U1908211 and 81641069).

\section{Footnote}

Conflicts of Interest: All authors have completed the ICMJE uniform disclosure form (available at http://dx.doi. org/10.21037/qims-20-501). ZS serves as an unpaid editorial board member of Quantitative Imaging in Medicine and Surgery. The other authors have no conflicts of interest to declare.

Ethical Statement: This study was approved by the animal care and use committee at the Capital Medical University of China.

Open Access Statement: This is an Open Access article distributed in accordance with the Creative Commons Attribution-NonCommercial-NoDerivs 4.0 International License (CC BY-NC-ND 4.0), which permits the noncommercial replication and distribution of the article with the strict proviso that no changes or edits are made and the original work is properly cited (including links to both the formal publication through the relevant DOI and the license). See: https://creativecommons.org/licenses/by-nc-nd/4.0/.

\section{References}

1. Hussain T, Dragulescu A, Benson L, Yoo SJ, Meng $\mathrm{H}$, Windram J, Wong D, Greiser A, Friedberg M, Mertens L, Seed M, Redington A, Grosse-Wortmann L. Quantification and significance of diffuse myocardial fibrosis and diastolic dysfunction in childhood hypertrophic cardiomyopathy. Pediatr Cardiol 2015;36:970-8.

2. Brouwer WP, Baars EN, Germans T, de Boer K, Beek AM, van der Velden J, van Rossum AC, Hofman MB. In- 
vivo T1 cardiovascular magnetic resonance study of diffuse myocardial fibrosis in hypertrophic cardiomyopathy. J Cardiovasc Magn Reson 2014;16:28.

3. Scully PR, Bastarrika G, Moon JC, Treibel TA. Myocardial Extracellular Volume Quantification by Cardiovascular Magnetic Resonance and Computed Tomography. Curr Cardiol Rep 2018;20:15.

4. Ma Q, Ma Y, Wang X, Li S, Yu T, Duan W, Wu J, Wen Z, Jiao Y, Sun Z, Hou Y. Circulating miR-1 as a potential predictor of left ventricular remodeling following acute ST-segment myocardial infarction using cardiac magnetic resonance. Quant Imaging Med Surg 2020;10:1490-503.

5. Radunski UK, Lund GK, Stehning C, Schnackenburg B, Bohnen S, Adam G, Blankenberg S, Muellerleile K. CMR in patients with severe myocarditis: diagnostic value of quantitative tissue markers including extracellular volume imaging. JACC Cardiovasc Imaging 2014;7:667-75.

6. Dall'Armellina E, Piechnik SK, Ferreira VM, Si QL, Robson MD, Francis JM, Cuculi F, Kharbanda RK, Banning AP, Choudhury RP, Karamitsos TD, Neubauer S. Cardiovascular magnetic resonance by non contrast T1-mapping allows assessment of severity of injury in acute myocardial infarction. J Cardiovasc Magn Reson 2012;14:15.

7. h-Ici DO, Jeuthe S, Al-Wakeel N, Berger F, Kuehne T, Kozerke S, Messroghli DR. T1 mapping in ischaemic heart disease. Eur Heart J Cardiovasc Imaging 2014;15:597-602.

8. Baggiano A, Boldrini M, Martinez-Naharro A, Kotecha T, Petrie A, Rezk T, Gritti M, Quarta C, Knight DS, Wechalekar AD, Lachmann HJ, Perlini S, Pontone G, Moon JC, Kellman P, Gillmore JD, Hawkins PN, Fontana $M$. Noncontrast Magnetic Resonance for the Diagnosis of Cardiac Amyloidosis. JACC Cardiovasc Imaging 2020;13:69-80.

9. Sado DM, White SK, Piechnik SK, Banypersad SM, Treibel T, Captur G, Fontana M, Maestrini V, Flett AS, Robson MD, Lachmann RH, Murphy E, Mehta A, Hughes D, Neubauer S, Elliott PM, Moon JC. Identification and assessment of Anderson-Fabry disease by cardiovascular magnetic resonance noncontrast myocardial T1 mapping. Circ Cardiovasc Imaging 2013;6:392-8.

10. Maron MS, Maron BJ, Harrigan C, Buros J, Gibson CM, Olivotto I, Biller L, Lesser JR, Udelson JE, Manning WJ, Appelbaum E. Hypertrophic cardiomyopathy phenotype revisited after 50 years with cardiovascular magnetic resonance. J Am Coll Cardiol 2009;54:220-8.

11. aus dem Siepen F, Buss SJ, Messroghli D, Andre F, Lossnitzer D, Seitz S, Keller M, Schnabel PA, Giannitsis
E, Korosoglou G, Katus HA, Steen H. T1 mapping in dilated cardiomyopathy with cardiac magnetic resonance: quantification of diffuse myocardial fibrosis and comparison with endomyocardial biopsy. Eur Heart J Cardiovasc Imaging 2015;16:210-6.

12. Flett AS, Hayward MP, Ashworth MT, Hansen MS, Taylor AM, Elliott PM, McGregor C, Moon JC. Equilibrium contrast cardiovascular magnetic resonance for the measurement of diffuse myocardial fibrosis: preliminary validation in humans. Circulation 2010;122:138-44.

13. Schelbert EB, Testa SM, Meier CG, Ceyrolles WJ, Levenson JE, Blair AJ, Kellman P, Jones BL, Ludwig DR, Schwartzman D, Shroff SG, Wong TC. Myocardial extravascular extracellular volume fraction measurement by gadolinium cardiovascular magnetic resonance in humans: slow infusion versus bolus. J Cardiovasc Magn Reson 2011;13:16.

14. Haaf P, Garg P, Messroghli DR, Broadbent DA, Greenwood JP, Plein S. Cardiac T1 Mapping and Extracellular Volume (ECV) in clinical practice: a comprehensive review. J Cardiovasc Magn Reson 2016;18:89.

15. Messroghli DR, Moon JC, Ferreira VM, GrosseWortmann L, He T, Kellman P, Mascherbauer J, Nezafat R, Salerno M, Schelbert EB, Taylor AJ, Thompson R, Ugander M, van Heeswijk RB, Friedrich MG. Clinical recommendations for cardiovascular magnetic resonance mapping of $\mathrm{T} 1, \mathrm{~T} 2, \mathrm{~T}^{*}$ and extracellular volume: A consensus statement by the Society for Cardiovascular Magnetic Resonance (SCMR) endorsed by the European Association for Cardiovascular Imaging (EACVI). J Cardiovasc Magn Reson 2017;19:75.

16. Kanter PM, Bullard GA, Ginsberg RA, Pilkiewicz FG, Mayer LD, Cullis PR, Pavelic ZP. Comparison of the cardiotoxic effects of liposomal doxorubicin (TLC D-99) versus free doxorubicin in beagle dogs. In Vivo 1993;7:17-26.

17. Arheden H, Saeed M, Higgins CB, Gao DW, Bremerich J, Wyttenbach R, Dae MW, Wendland MF. Measurement of the distribution volume of gadopentetate dimeglumine at echo-planar MR imaging to quantify myocardial infarction: comparison with 99mTc-DTPA autoradiography in rats. Radiology 1999;211:698-708.

18. Fullerton GD, Potter JL, Dornbluth NC. NMR relaxation of protons in tissues and other macromolecular water solutions. Magn Reson Imaging 1982;1:209-26.

19. Martin MA, Tatton WG, Lemaire C, Armstrong RL. Determination of extracellular/intracellular fluid ratios 
from magnetic resonance images: accuracy, feasibility, and implementation. Magn Reson Med 1990;15:58-69.

20. Li W, Grgac K, Huang A, Yadav N, Qin Q, van Zijl PC. Quantitative theory for the longitudinal relaxation time of blood water. Magn Reson Med 2016;76:270-81.

21. Messroghli DR, Walters K, Plein S, Sparrow P, Friedrich MG, Ridgway JP, Sivananthan MU. Myocardial T1 mapping: application to patients with acute and chronic myocardial infarction. Magn Reson Med 2007;58:34-40.

22. Messroghli DR, Niendorf T, Schulz-Menger J, Dietz R, Friedrich MG. T1 mapping in patients with acute myocardial infarction. J Cardiovasc Magn Reson 2003;5:353-9.

23. Sparrow P, Messroghli DR, Reid S, Ridgway JP, Bainbridge G, Sivananthan MU. Myocardial T1 mapping for detection of left ventricular myocardial fibrosis in chronic aortic regurgitation: pilot study. AJR Am J Roentgenol 2006;187:W630-5.

24. Iles L, Pfluger H, Phrommintikul A, Cherayath J, Aksit P, Gupta SN, Kaye DM, Taylor AJ. Evaluation of diffuse myocardial fibrosis in heart failure with cardiac magnetic resonance contrast-enhanced T1 mapping. J Am Coll Cardiol 2008;52:1574-80.

25. Han Y, Peters DC, Dokhan B, Manning WJ. Shorter difference between myocardium and blood optimal inversion time suggests diffuse fibrosis in dilated cardiomyopathy. J Magn Reson Imaging 2009;30:967-72.

26. Amano Y, Takayama M, Kumita S. Contrast-enhanced myocardial T1-weighted scout (Look-Locker) imaging for the detection of myocardial damages in hypertrophic cardiomyopathy. J Magn Reson Imaging 2009;30:778-84.

27. Sado DM, Flett AS, Banypersad SM, White SK, Maestrini V, Quarta G, Lachmann RH, Murphy E, Mehta A, Hughes DA, McKenna WJ, Taylor AM, Hausenloy DJ, Hawkins
PN, Elliott PM, Moon JC. Cardiovascular magnetic resonance measurement of myocardial extracellular volume in health and disease. Heart 2012;98:1436-41.

28. Treibel TA, Fontana M, Maestrini V, Castelletti S, Rosmini S, Simpson J, Nasis A, Bhuva AN, Bulluck H, Abdel-Gadir A, White SK, Manisty C, Spottiswoode BS, Wong TC, Piechnik SK, Kellman P, Robson MD, Schelbert EB, Moon JC. Automatic Measurement of the Myocardial Interstitium: Synthetic Extracellular Volume Quantification Without Hematocrit Sampling. JACC Cardiovasc Imaging 2016;9:54-63.

29. Robison S, Karur GR, Wald RM, Thavendiranathan P, Crean AM, Hanneman K. Noninvasive hematocrit assessment for cardiovascular magnetic resonance extracellular volume quantification using a point-of-care device and synthetic derivation. J Cardiovasc Magn Reson 2018;20:19.

30. Narayan HK, Wei W, Feng Z, Lenihan D, Plappert T, Englefield V, Fisch M, Ky B. Cardiac mechanics and dysfunction with anthracyclines in the community: results from the PREDICT study. Open Heart 2017;4:e000524.

31. Shang Y, Zhang X, Zhou X, Wang J. Extracellular volume fraction measurements derived from the longitudinal relaxation of blood-based synthetic hematocrit may lead to clinical errors in $3 \mathrm{~T}$ cardiovascular magnetic resonance. $\mathrm{J}$ Cardiovasc Magn Reson 2018;20:56.

32. Raucci FJ Jr, Parra DA, Christensen JT, Hernandez LE, Markham LW, Xu M, Slaughter JC, Soslow JH. Synthetic hematocrit derived from the longitudinal relaxation of blood can lead to clinically significant errors in measurement of extracellular volume fraction in pediatric and young adult patients. J Cardiovasc Magn Reson 2017;19:58.
Cite this article as: Zhou Z, Wang R, Wang H, Liu Y, Lu D, Sun Z, Yang G, Xu L. Myocardial extracellular volume fraction quantification in an animal model of the doxorubicin-induced myocardial fibrosis: a synthetic hematocrit method using $3 \mathrm{~T}$ cardiac magnetic resonance. Quant Imaging Med Surg 2021;11(2):510-520. doi: 10.21037/qims-20-501 